Journal of Computer Science 7 (3): 440-447, 2011

ISSN 1549-3636

(C) 2011 Science Publications

\title{
Enhanced Clustering Techniques for Hyper Network Planning using Minimum Spanning Trees and Ant-Colony Algorithm
}

\author{
${ }^{1,2}$ Lamiaa Fattouh Ibrahim \\ ${ }^{1}$ Department of Computer Sciences and Information, \\ Institute of Statistical Studies and Research, Cairo University, Cairo, Egypt \\ ${ }^{2}$ Department of Information Technology, \\ Faculty of Computing and Information Technology, King AbdulAziz University, \\ B.P. 42808 Zip Code 21551- Girl Section, Jeddah, Saudi Arabia
}

\begin{abstract}
Problem statement: The process of network planning is divided into two sub steps. The first step is determining the location of the Multi Service Access Node (MSAN). The second step is the construction of subscriber network lines from MSAN to subscribers to satisfy optimization criteria and design constraints. Due to the complexity of this process artificial intelligence and clustering techniques have been successfully deployed to solve many problems. The problems of the locations of MSAN, the cabling layout and the computation of optimum cable network layouts have been addressed in this study. The proposed algorithm, Clustering density-Based Spatial of Applications with Noise original, minimal Spanning tree and modified Ant-Colony-Based algorithm (CBSCAN-SPANT), used two clustering algorithms which are density-based and agglomerative clustering algorithm using distances which are shortest paths distance and satisfying the network constraints. This algorithm used wire and wireless technology to serve the subscribers demand and place the switches in a real optimal place. Approach: The density-based Spatial Clustering of Applications with Noise original (DBSCAN) algorithm has been modified and a new algorithm (NetPlan algorithm) has been proposed by the author in a recent work to solve the first step in the problem of network planning. In the present study, the NetPlan algorithm is modified by introduce the modified Ant-Colony-Based algorithm to find the optimal path between any node and the corresponding MSAN node in the first step of network planning process to determine nodes belonging to each cluster. The second step, in the process of network planning, is also introduced in the present study. For each cluster, the optimal cabling layout from each MSAN to the subscriber premises is determining by introduce the Prime algorithm which construct minimal spanning tree. Results: Experimental results and analysis indicate that the CBSCAN-SP-ANT algorithm was effective, leads to minimum costs for network construction and make the best grade of service. Conclusion: Using mobile network to serve the area with low density is decreasing the cost of design the fixed wire network. Also, using the modified ANT algorithm and minimum spanning tree, are helping to construct the cable layout from each MSAN to subscribers when the network is complicated and the number of intersections and streets are large.
\end{abstract}

Key words: Multi Service Access Node (MSAN), agglomerative algorithm, clustering techniques, network planning, cable layout network, short path, Minimum Spanning Tree (MST), ant-colony algorithm, meta-heuristic, NetPlan algorithm

\section{INTRODUCTION}

The goal of a clustering algorithm is to partition a given data set into clusters or groups, which are not predefined, such that the data points in a cluster are similar to each other more than points in different clusters. These groups are formed according to some measures of goodness that differ according to application.

The field of "ant algorithms" studies models derived from the observation of real ant's behavior and uses these models as a source of inspiration for the design of novel algorithms for solution of optimization and distributed control problems (Dorigo and Stulzle, 2004; Gunes et al., 2002; Othman et al., 2007; ALSalami, 2009).

Ant colony algorithms are a subset of swarm intelligence and consider the ability of simple ants to solve complex problems by cooperation. The interesting point is, that the ants do not need any direct communication for the solution process, instead they 
communicate by stigmergy. The notion of stigmergy means the indirect communication of individuals through modifying their environment. Several algorithms which are based on ant colony problems were introduced in recent years to solve different problems, e.g., optimization problems, image segmentation (Ouadfel and Batouche, 2007; Ibrahim et al., 2005; Hashim and Abdl, 2010).

Multi Service Access Node MSAN is one of the dominant access delivery methods and comes in modular units which may be equipped with line cards supporting a number of services, differing capacities, ranging from a few dozen lines up to thousands of lines.

In many countries, there is tremendous demand for new business and residential telephone service. The continuous Increasing of number of subscribers make congestion in MSAN and cause degradation in grade of service and in sometimes impossible to add new subscribers which lead to using the mobile tower. Also, the existing of the natural obstacle is affecting on distribution the MSAN on the regions.

In this study we determine place of each MSAN using NetPlan algorithm (Ibrahim et al., 2009) after modified by introduce the modified Ant-Colony-Based algorithm to find the optimal path between any node and the corresponding MSAN node in the first step of network planning process to determine nodes belong to each cluster. When the congestion in MSAN is occur mobile tower is playing as auxiliary tool with maximum 100 subscribers.

The second step is also introduced in the present paper. For each cluster, the optimal cabling layout from each MSAN to the subscriber premises must be determined. The problem is to find the least cost network by minimizing the distances from the MSAN to the subscribers belonging to it. Thus the problem of connecting $\mathrm{n}$ nodes with a minimum distance network is the problem of finding a minimal spanning tree in a connected weighted graph of $n$ vertices. The Prime algorithm is used to construct the optimal cabling layout for each cluster. The final step is the calculation of total cost.

Due to the complexity of this process, Artificial Intelligence (AI) (Fahmy and Douligeris, 1997; ElDessouki et al., 1999) and partitioning clustering techniques (Khaled et al., 2003; Ibrahim and Al Harbi, 2008a; 2008b; Harby and Ibrahim, 2008; Ibrahim, 2005; 2006; Han et al., 2001) successfully deployed in a number of areas.

The NetPlan algorithm: Network Planning package (NetPlan) (Ibrahim et al., 2009) is divided into two steps:
Step 1: Applying the modified DBSCAN algorithm.

Step 2: Applying Agglomerate algorithm to the resulted clusters.

Modified DBSCAN algorithm: Two parameters must me determine before we starts applying the DBSCAN. These parameters are MinPts and Eps. In network planning the cable length must be at maximum $2.5 \mathrm{~km}$ for $0.4 \mathrm{~cm}$ diameters to achieve an acceptable grade of service. So, we make the value of EPS take the value of shortest path from core (MSAN) to the most remote point (subscribers) which is $2.5 \mathrm{~km}$. The original DBSCAN Algorithm uses Euclidian distance (that means the direct distance between the MSAN and nodes); The Direct Euclidean distance ignores the presence of streets and paths that must be taken into consideration during clustering. In NetPlan, a clustering based solution is presented depending on using the physical shortest available routes. To apply shortest path we selected Dikjstra algorithm.

When the congestion in MSAN is occur or the number of subscribers is less than 100 we use mobile tower as auxiliary tool to serve this small number of subscribers. Therefore the value of MinPts is set to 101 .

The DBSCAN classify nodes to:

- Core point which is a subset of candidate MSAN location

- Noise point: In real planning all subscribers must be served so noise point is served using the mobile tower which can serve at maximum 100 subscribers because that number is the maximum number of subscriber who can be served by mobile tower

- Border point that belong to ascertain cluster

NetPlan use Dijkstra algorithm to calculate shortest path from one node to all MSANs (the reason is to determine the nearest suitable MSAN that will serve this node).

Agglomerative clustering technique: The agglomerative clustering technique is hierarchal clustering technique. It starts with the points as individual clusters and at each step merge the closest pair of clusters depends on a notion of cluster proximity and the faraway node from MSAN which should be at maximum $2.5 \mathrm{~km}$ to achieve the required grade of service. In NetPlan, after we distribute the nodes into different clusters (each cluster has served by one MSAN) and maybe there are two cores (MSANs) are close in distance (less than $1.25 \mathrm{Km}$ ). So, we need to decrease the cost of constructing a new MSAN if one of them can hold the loads (subscribers) of the two MSANs. Figure 1 shows Pseudo code of agglomerative algorithm used in NetPlan. 
Construct the finest partition (clusters).

Compute the distance matrix.

DO

Find the two clusters with the closest distance.

Put those two clusters into one cluster if the distance condition is satisfied.

Compute the distance between the new groups and obtain a reduced distance matrix

UNTIL all possible clusters are agglomerated.

Fig. 1: Pseudo code of agglomerative algorithm

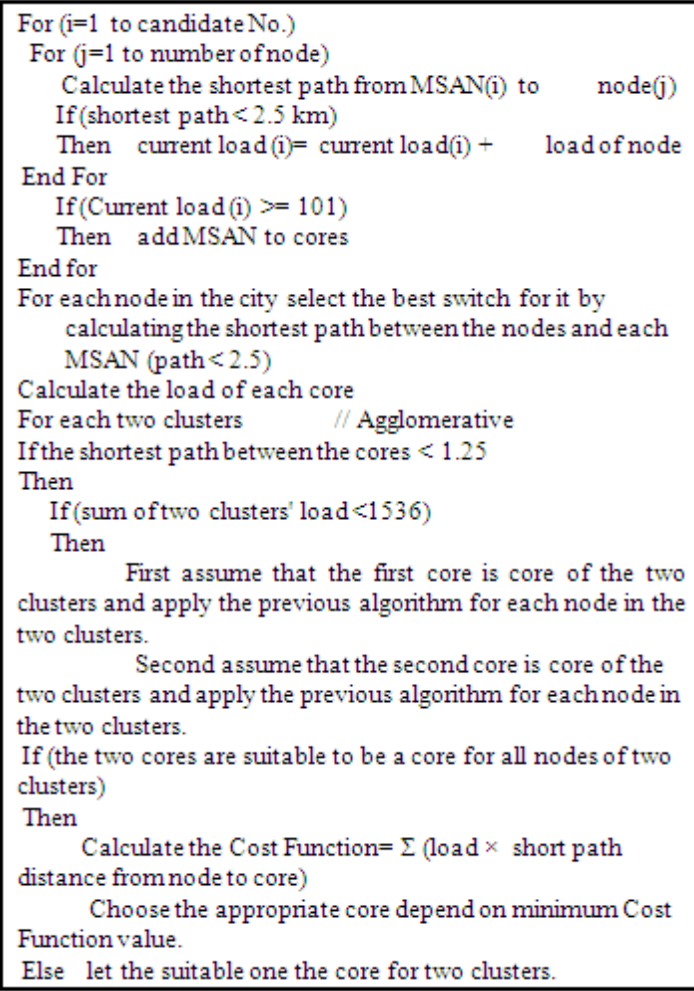

Fig. 2: NetPlan algorithm

Implemented of NetPlan algorithm: The user first inserts the location of candidate MSAN. NetPlan uses these candidate locations as candidate core points and chooses the one which satisfies the condition to be a core node. After this step the NetPlan determine the boundaries of cluster by calculate the short path from node to each core and allocate the node to the minimum short path core. The following step is the agglomerative step with mix two clusters if the overall load is less than the max load of MSAN which is 1536 and if also the maximum short path for all node to core is less or equal $2.5 \mathrm{~km}$ and the third condition if the distance between the two core is less the half of $2.5 \mathrm{~km}$. NetPlan introduces a cost function which is the sum of multiplication of load of node and short path between node and core. This cost function is used to choose the best MSAN when we merge two clusters. Figure 2 shows pseudo code of NetPlan algorithm used.

\section{MATERIALS AND METHODS}

The proposed algorithm CBSCAN-SP-ANT modified NetPlan algorithm by introduce the modified Ant-Colony-Based algorithm to find the optimal path between any node and the corresponding MSAN node in the first step of network planning process to determine nodes belong to each cluster. The second step, in the process of network planning, is also introduced in the present study. For each cluster, the optimal cabling layout from each MSAN to the subscriber premises is determining by introduce the Prime algorithm which construct minimal spanning tree.

The problem statement:

- Input: A set $\mathrm{P}$ data points $\left\{\mathrm{p}_{1}, \mathrm{p}_{2} \ldots \mathrm{p}_{\mathrm{n}}\right\}$ in 2-D map which represent intersection nodes, coordinates of each node, a map of streets, distribution of the subscribers' loads within the city and the location of base station in mobile network in this city

- The available cable sizes, the cost per unit for each size and the maximum distance of wire that satisfied the allowed grade of service

- Objective: Partition the city into $\mathrm{k}$ clusters $\left\{\mathrm{C}_{1}, \mathrm{C}_{2}\right.$, .., $\left.\mathrm{C}_{\mathrm{k}}\right\}$ that satisfy clustering constraints, such that the cost function is minimized with high grade of services

- Output: $\mathrm{k}$ clusters, the location of MSAN, the cable layout from each MSAN to subscriber, boundaries of each cluster and finally the total cost to construct the network

Computing the shortest distance from node to MSAN: The ANT colony optimization meta-heuristic is a particular class of ant algorithms. Ant algorithms are multi-agent systems, which consist of agents with the behavior of individual ant (Bonabeau et al., 1999; Wittner and Helvik, 2004).

The basic idea of the ant-colony optimization metaheuristic is taken from the food searching behavior of real ants. When ants are on the way to search for food, they start from their nest and walk toward the food. When an ant reaches an intersection, it has to decide which branch to take next. While walking, ants deposit pheromone, which marks the route taken. The concentration of pheromone on a certain path is an 
indication of its usage. With time the concentration of pheromone decreases due to diffusion effects.

All ants take the shortest path after an initial searching time.

The Ant colony algorithm depends mainly on two steps as follows:

- Tour construction

- Pheromone update

Tour construction: At each construction step, the probability with which ant $\mathrm{k}$, currently at node i, chooses to go to node $\mathrm{j}$ at the $\mathrm{t}^{\text {th }}$ iteration of the algorithm is:

$P_{i j}^{k}(t)=\frac{\left[T_{i j}(t)\right]^{\alpha} \cdot\left[\eta_{i j}\right]^{\beta}}{\sum_{i \in N_{i}^{k}}\left[T_{i j}(t)\right]^{\alpha} \cdot\left[\eta_{i j}\right]^{\beta}}$

If $\mathrm{j} \in \mathrm{N}_{\mathrm{ik}}$ and 0 otherwise

Where:

$\eta_{\mathrm{ij}}=1 / \mathrm{d}_{\mathrm{ij}}$ is an a priori available heuristic value. It is known as visibility of node $\mathrm{j}$ from node $\mathrm{i}$

$\mathrm{T}_{\mathrm{ij}}=$ Intensity of pheromone trail between node $\mathrm{i}$ and node $\mathrm{j}$

$\mathrm{N}_{\mathrm{i}}^{\mathrm{j}}=$ The set of nodes which ant $\mathrm{k}$ has not yet visited

$\alpha=p \$ a$ arameter to regulate the influence of $T_{i j}$

$\beta=$ Parameter to regulate the influence of $\eta_{i j}$

$\mathrm{d}_{\mathrm{ij}}=$ Distance between node $\mathrm{i}$ and node $\mathrm{j}$ ( in case of shortest path problem)

Pheromone update: After all ants have constructed their tours, the pheromone trails are updated:

$$
T_{i j}(t+1)=(1-p) \cdot T_{i j}(t)+\sum_{k=1}^{m} \Delta T_{i j}^{k}(t)
$$

where, $0<\mathrm{p} \leq 1$ is the pheromone trail evaporation.

$\Delta \mathrm{T}_{\mathrm{ij}}{ }^{\mathrm{k}}(\mathrm{t})$ is the amount of pheromone which ant $\mathrm{k}$ puts on the arcs it has visited; it is defined as follows:

$$
\Delta \mathrm{T}_{\mathrm{ij}}^{\mathrm{k}}(\mathrm{t})=\left\{\begin{array}{cc}
1 / \mathrm{L}^{\mathrm{k}}(\mathrm{t}) & \text { if aro }(\mathrm{i}, \mathrm{j}) \text { is used by ant } \mathrm{k} \\
0 & \text { otherwise }
\end{array}\right.
$$

where, $L^{k}(t)$ is the total length of the tour, from source to destination, made by the ant $\mathrm{k}$ at iteration $\mathrm{t}$. By the last equation, the better the ant's tour is, the more pheromone is received by arcs belonging to the tour. Figure 3 shows the fundamental Algorithm of Ant Algorithm.

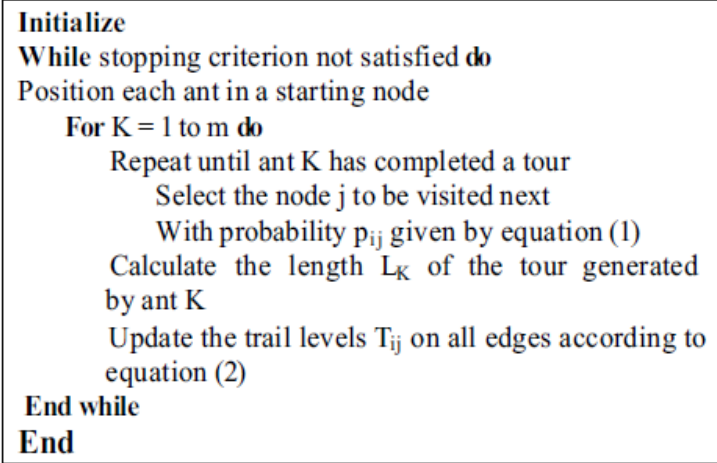

Fig. 3: Fundamental algorithm of ant algorithm

The ANP Ant-Colony-Based Network Planning algorithm (Ibrahim et al., 2005) modified the pervious describe algorithm in Fig. 3 due to a number of problems are raised when we try to apply this algorithm such as:

- Loss of ants due to some node is connected with the network by terminates edge and the ant is not permitted to visit node twice

- Ant is blocked when it reaches terminates node and has no way to reach the location of the switch

- Loss of ants due to internal loop in a graph

Figure 4 shows ANP Ant-Colony-Based Network Planning Algorithm. In This algorithm, for each node we put a number of ants equal the number of edges (no ants at switch location) to substitute the losses of ants and at the first step we move each ant at different route edge.

Optimal cabling layout: For each cluster, the optimal cabling layout from each exchange to the subscriber premises must be determined. The problem is to find the least cost network by minimizing the distances from the exchange to the subscribers belonging to it. Thus the problem of connecting $\mathrm{n}$ nodes with a minimum distance network is the problem of finding a minimal spanning tree in a connected weighted graph of $n$ vertices. The Prime algorithm is used to construct the optimal cabling layout for each cluster.

The Prime algorithm (O'Rourke, 1994) is used to grow the minimum spanning tree from a root (core) to find the least expensive network that connects all nodes together. The Minimum Spanning Tree (MST) is a tree that connects all nodes in the cluster with minimum cost and achieves the maximum grade of service. Figure 5 shows Prime algorithm.

Figure 6 shows pseudo code of CBSCAN-SP-ANT Algorithm used. 


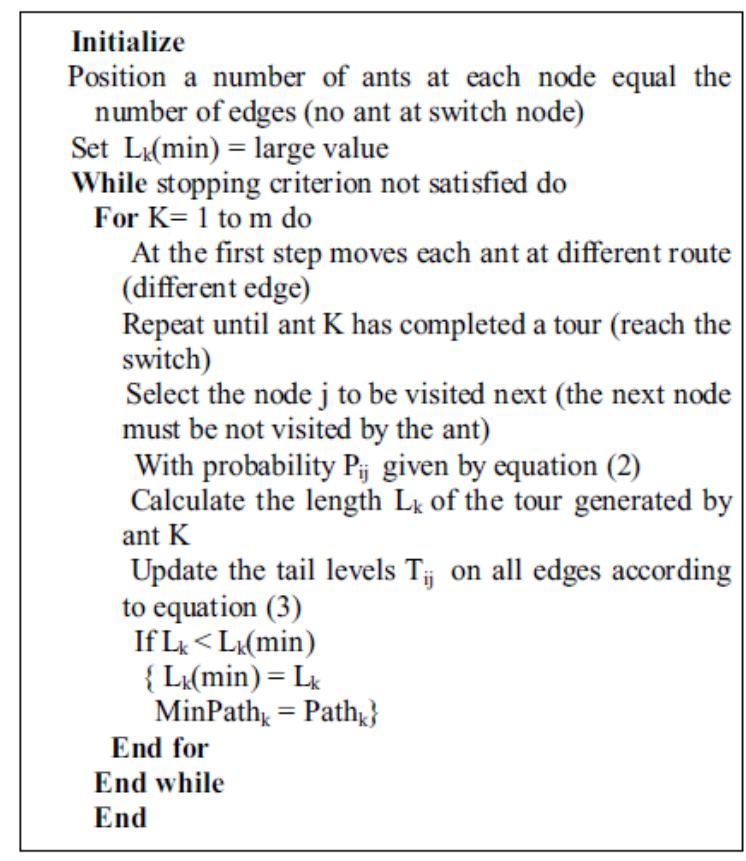

Fig. 4: ANP ant-colony-based network planning algorithm

\begin{tabular}{|c|}
\hline $\begin{array}{l}\text { Algorithm } 3 \text { Prime } \\
\text { Input: nodes, root (core), list of weights. } \\
\text { Output: minimum spanning tree (MST). } \\
\text { Method }\end{array}$ \\
\hline 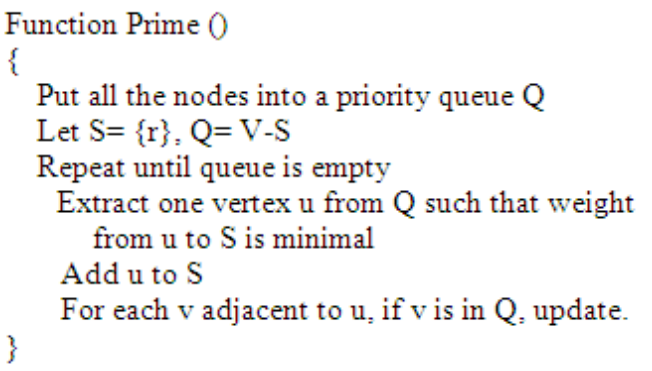 \\
\hline
\end{tabular}

Fig. 5: Prime algorithm

\section{RESULTS AND DISCUSSION}

Table 1 compares related study. In Gravity Center algorithm (Dessouki et al., 1999), the city is divided into four quadrants (clusters) at the center of gravity if the capacity is more than the capacity of max switch capacity allow or distance from node to switch is more than the max distance allow. The switches will be located at the center of gravity of each cluster. If the constraints are not satisfied in any of the four quadrants

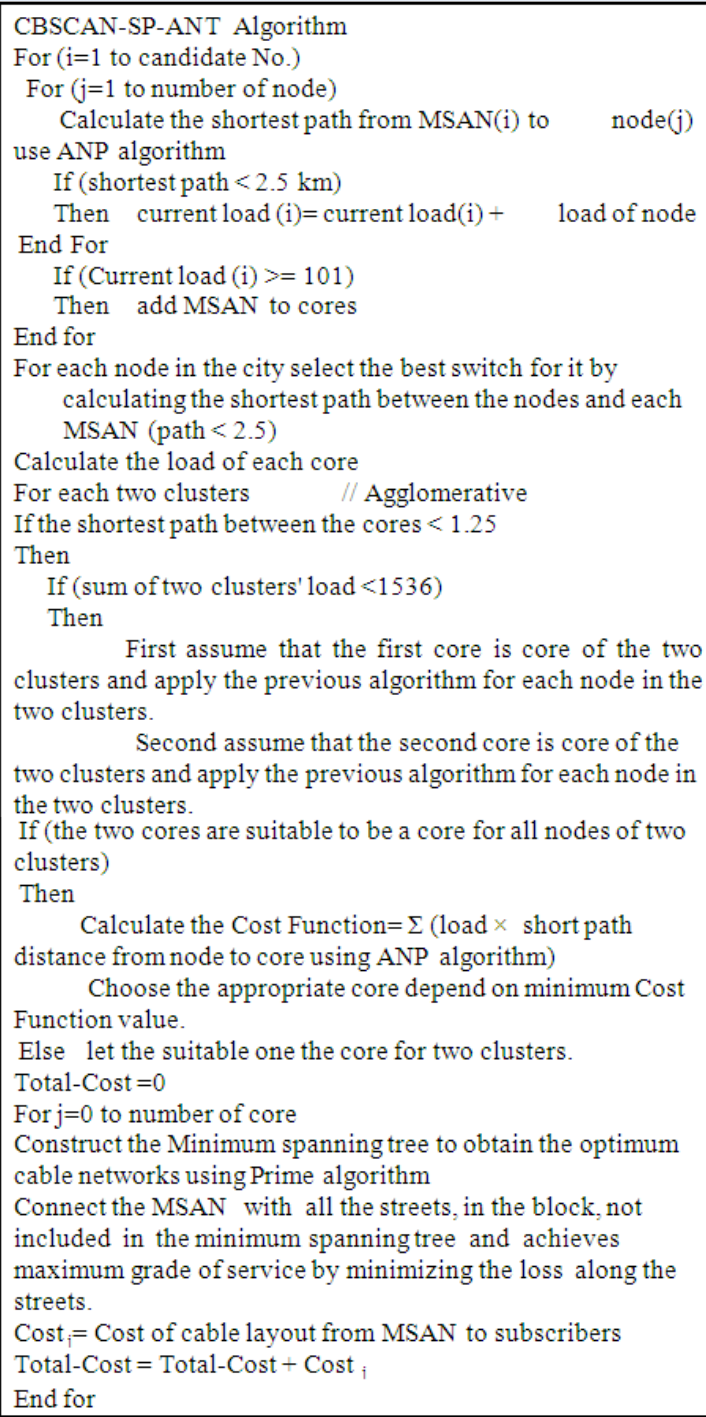

Fig. 6: CBSCAN-SP-ANT algorithm

the same partitioning method is applied to the quadrant which does not satisfy the constraints. This yields that the number of clusters equal seven partitions. This method will be iterated until the network constraints are 7,10 . This study doesn't reflect the real nature of the clusters, or the number of the suitable clusters, it is always incrementing the number of clusters by three. COD-CLARANS (Tung et al., 2001) and CSPwCLARANS (El-Dessouki et al., 1999) algorithms depend mainly on CLARANS which is design to deal with large database by using multiple different samples. These two algorithms is very powerfully when we plan a large city, but not acute when we plan small city due the sampling use. 
J. Computer Sci., 7 (3): 440-447, 2011

Table 1: A comparison between related works

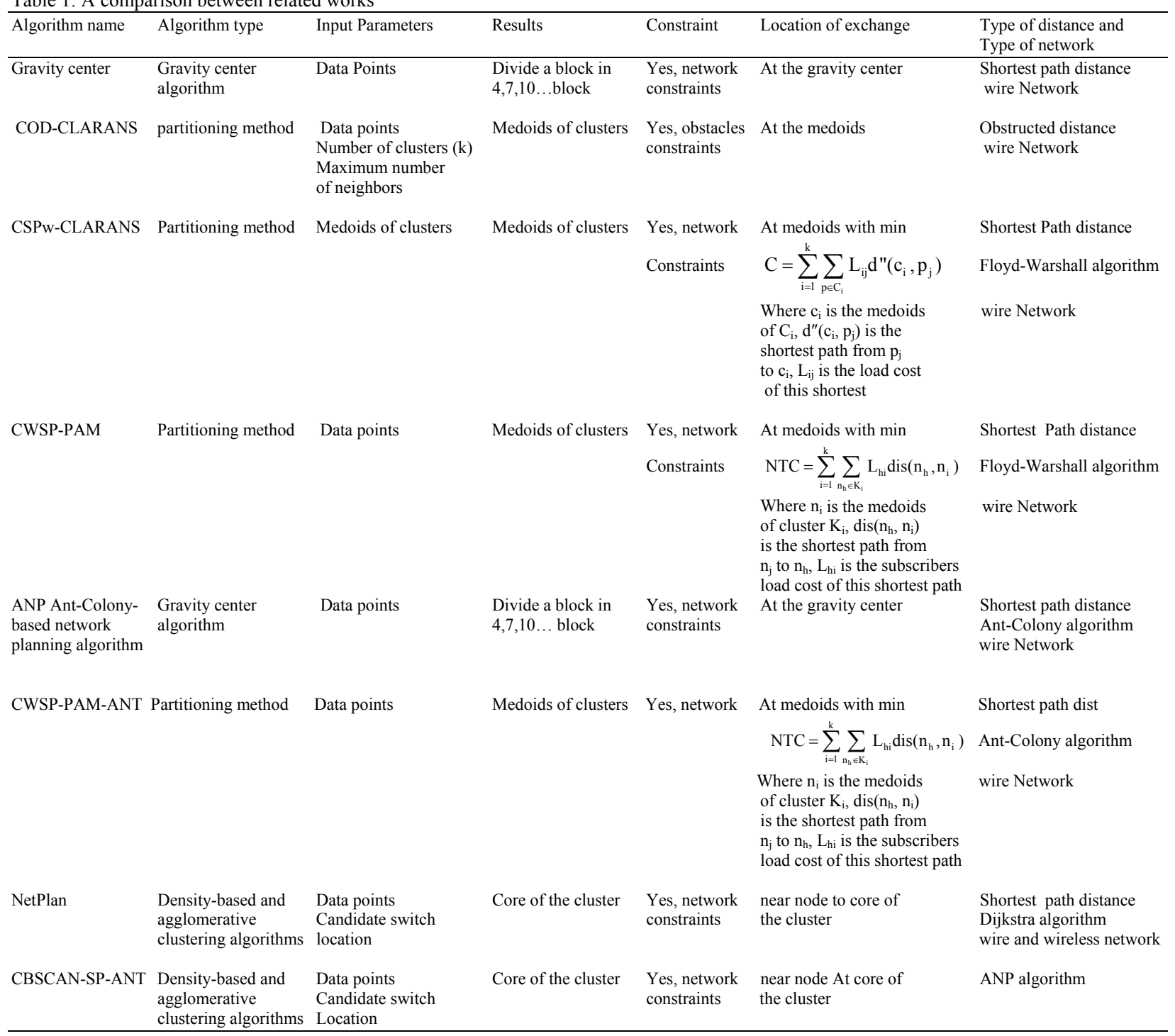

satisfied. The resulting number of clusters may be 1,4 , ant-colony-based network planning algorithm (Ibrahim et al., 2005) used Gravity center to find the location of switch and applied a modified version of Ant-colony algorithm to find the shortest path. The algorithm is very powerful when the network is complicated and we have a large number of intersection and streets but has the disadvantages of Gravity center algorithm mention above.

CWSP-PAM (Ibrahim, 2005) algorithm depends mainly on PAM clustering algorithm. This algorithm use Floyd-Warshall algorithm to find short path.

The CWSP-PAM-ANT (Ibrahim, 2006) (Clustering with Shortest Path-PAM and ANT-Colony algorithms) modified the CWSP-PAM algorithm by introduce the Ant-Colony-Based algorithm to find the optimal path between any node and the corresponding switch node.

The NetPlan algorithm used two clustering algorithms which are density-based and agglomerative clustering algorithm using distances which are shortest paths distance calculated by Dijkstra algorithm and satisfying the network constraints. This algorithm uses wire and wireless technology to serve the subscribers demand and place the switches in a real place which are determined by the candidate locations entered by the user of the package. This algorithm has two disadvantages. First, Dijkstra algorithm fail when the network is complicated and we have a large number of intersection and streets. The second disadvantage, the algorithm does not determine the cable layout from MSAN to subscriber and does not calculate the total cost. 


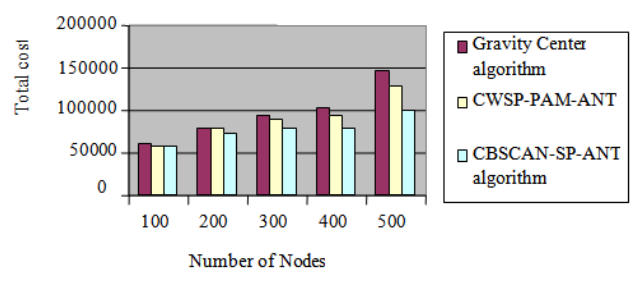

Fig. 7: Cost comparisons between gravity center and CBSCAN-SP-ANT algorithms

Figure 7 Compare between the total cost calculated by Gravity Center algorithm, CWSP-PAM-ANT and CBSCAN-SP-ANT algorithm when the database size is increased gradually to show the better result of CBSCAN-SP-ANT algorithm against Gravity Center algorithm and CWSP-PAM-ANT algorithm. The figure showed the decrease of cost when used CBSCAN-SPANT where the Gravity Center algorithm has the largest number of cluster which mean the largest number of switch and largest cost. In CBSCAN-SPANT algorithm the number of MSAN is smallest due to the use of mobile network when a small number of subscribers are existed in non density areas and the use of agglomerative clustering algorithms to merge small clusters where the CWSP-PAM-ANT algorithm introduce MSAN in any constructed cluster even if the number of subscribers is small (less than 100 subscribers) which increase the total cost.

\section{CONCLUSION}

Clustering analysis is one of the major tasks in various research areas. The clustering aims at identifying and extracting significant groups in underlying data. Based on certain clustering criteria the data are grouped so that the data points in a cluster are more similar to each other than points in different clusters. Ant colony algorithms are a subset of swarm intelligence and consider the ability of simple ants to solve complex problems by cooperation. In this study, we have studied the problem of network planning and the construction of optimal cable network layouts. The algorithm CBSCAN-SP-ANT algorithm is introduced to solve this problem. This algorithm used two clustering algorithms which are density-based and agglomerative clustering algorithm using shortest paths calculated by ANP algorithm, which is a modified of ant colony algorithm and also used Minimum spanning algorithm, Prime algorithm, to construct the cable layout and satisfying the network constraints. This algorithm uses wire and wireless technology to serve the subscribers demand and place the switches in a real place which are determined by the candidate locations entered by the user of the package. Experimental results and analysis indicate that CBSCAN-SP-ANT algorithm leads to minimum costs for network construction in an area where accuracy is needed and the network is complicated and we have a large number of intersections and streets.

It is expected that by applying this system to a number of areas belonging to different countries with different sizes, one can verify its capabilities more universally.

Another objective, which is currently under consideration, is to extend the present work to design a mobile network. Such networks have others complex constraints and varies of equipments.

\section{REFERENCES}

AL-Salami, N.M.A., 2009. System evolving using ant colony optimization algorithm. J. Comput. Sci., 5: 380-387. DOI: 10.3844 jessp.2009.380.387

Bonabeau, E., M. Dorigo and G. Theraulaz, 1999. Swarm Intelligence: From Natural to Artificial Intelligence. 1st Edn., Oxford University Press, Pakistan, ISBN-10: 0195131584, pp: 307.

Dorigo, M. and T. Stulzle, 2004. Ant Colony Optimization. 1st Edn., The MIT Press, United States, ISBN-10: 0262042193, pp: 319.

El-Dessouki, A., A.E.M. Wahdan and L.F. Ibrahim, 1999. The use of knowledge-based system for urban telephone planning. Proceedings of the International Telecommunication Union, ITU/ITC/LAS Regional Seminar on Tele-Traffic Engineering for Arab States, Nov. 7-12, Damascus. http://d.yimg.com/kq/groups/24142996/207155487 5/name/paper

Fahmy, H.I. and C. Douligeris, 1997. Application of neural networks and machine learning in network design. IEEE J. Selected Areas Commun., 15: 226237. DOI: $10.1109 / 49.552072$

Gunes, M., U. Sorges and I. Bouazizi, 2002. ARA-the ant-colony based routing algorithm for MANETs. Proceeding of the International Conference on Parallel Processing Workshops, (ICPPW'02), Aachen University of Technology, Germany, pp: 79-85. DOI: 10.1109/ICPPW.2002.1039715

Han, J., M. Kamber and A.K.H. Tung, 2001. Spatial clustering methods in data mining: A survey. In: Geographic data mining and knowledge discovery, Miller, H.J. and J. Han (Eds.). Taylor and Francis, London, pp: 188-217. 
Harby, M.E. and L.F. Ibrahim, 2008. Employing of clustering algorithm CWN-PAM in mobile network planning. Proceedings of the 3rd International Conference on Systems and Networks Communications, Oct. 26-31, Sliema, Malta, pp: 44-49. DOI: 10.1109/ICSNC.2008.71

Hashim, S.Z.M. and K.M.B. Abdl, 2010. Swarm-based feature selection for handwriting identification. J. Comput. Sci., 6: 80-86. DOI: 10.3844/jcssp.2010.80.86

Ibrahim, L.F. and M.H. Al Harbi, 2008a. Employing clustering techniques in mobile network planning. Proceedings of the 2nd IFIP International Conference on New Technologies, Mobility and Security, Nov. 5-7, Tangier, Morocco, pp: 1-9. DOI: 10.1109/NTMS.2008.ECP.42

Ibrahim, L.F. and M.H. Al Harbi, 2008b. Using clustering technique M-PAM in mobile network planning. Proceedings of the 12th WSEAS International Conference on COMPUTERS, July 23-25, Heraklion, Crete Island, Greece, pp: 868-873.

Ibrahim, L.F., 2005. Using of clustering algorithm CWSP-PAM for rural network planning. Proceedings of the 3rd International Conference on Information Technology and Applications, July 47, Sydney, Australia, pp: 280-283. DOI: 10.1109/ICITA.2005.300

Ibrahim, L.F., 2006. Using of clustering and ant-colony algorithms CWSP-PAM-ANT in network planning. Proceedings of the International Conference on Digital Telecommunications, Aug. 26-31, Cote d'Azur, pp: 63-63. DOI: 10.1109/ICDT.2006.77

Ibrahim, L.F., O. Metwaly, A. Kabil and A. Abid, 2005. Enhancing the behavior of the ant algorithms to solving network planning problem. Proceedings of the 3rd ACIS International Conference on Software Engineering, Research, Management and Applications, Aug. 11-13, Mt. Pleasant, Michigan, USA., pp: 250-255.
Ibrahim, L.F., W.M. Minshawi, I.Y. Ekkab, N.M. AL-Jurf and A.S. Babrahim et al., 2009. Enhancing the DBSCAN and agglomerative clustering algorithms to solve network planning problem. Proceedings of the International Conference on Data Mining Workshops, Dec. 6-6, Miami, Florida, USA., pp: 662-667. DOI: 10.1109/ICDMW.2009.98

Khaled, W., O. Karam, L. Fattouh and M.A. El Sharkawy, 2003. CSPw-CLARANS: An enhanced clustering technique for network planning using minimum spanning trees. Proceedings of the International Arab Conference on Information Technology, Dec. 20-23, Alexandri, Egypt. http://www.shams.edu.eg/cis/is/cv/walaakhaled.pdf

O'Rourke, J., 1994. Computational Geometry in C. 2nd Edn., Cambridge University Press, Cambridge, UK., ISBN-10: 0521649765, pp: 376.

Othman, Z.A., H.M. Rais and A.R. Hamdan, 2007. Embedding Malaysian house red ant behavior into an ant colony system. J. Comput. Sci., 4: 934-941. DOI: 10.3844 jessp.2008.934.941

Ouadfel, S. and M. Batouche, 2007. An efficient ant algorithm for swarm-based image clustering. J. Comput. Sci., 3: 162-167. DOI: 10.3844/jcssp.2007.162.167

Tung, A.K.H., J. Hou and J. Han, 2001. Spatial clustering in the presence of obstacles. Proceedings of the 17th International Conference on Data Engineering, Apr.02-06, Heidelberg, Germany, pp: 359-367. DOI: 10.1109/ICDE.2001.914848

Wittner, O. and B.E. Helvik, 2004. Distributed soft policy enforcement by swarm intelligence; application to loadsharing and protection. Annals Telecommun., 59: 10-24. DOI: 10.1007/BF03179671 